\title{
Midterm clinical and radiographic results of the medial pivot total knee system
}

\author{
Robert Schmidt • Steven Ogden • J. David Blaha • \\ Angelia Alexander • David A. Fitch • C. Lowry Barnes
}

Received: 9 June 2014 / Accepted: 21 June 2014 / Published online: 11 July 2014

(C) The Author(s) 2014. This article is published with open access at Springerlink.com

\begin{abstract}
Purpose The ADVANCE ${ }^{\circledR}$ Medial Pivot Knee System was designed with a highly congruent medial compartment and a less conforming lateral compartment to more closely mimic the kinematics of the normal knee. The purpose of this study was to evaluate the midterm clinical and radiographic outcomes of this total knee arthroplasty (TKA) system.

Methods Between January 1998 and December 2006, 421 primary TKAs were performed in 373 subjects using this system and a surgical technique that resects the posterior cruciate ligament. Of these, 365 TKAs in 320 subjects were available for a follow-up visit occurring at a mean of 5.3 years. Subjects were evaluated using Knee Society Scores, range of motion, and radiographic review.

Results The average Knee Society clinical score was 95.5 at final follow-up, with 358 (98\%) TKAs having excellent or good results. Range of motion increased from a preoperative mean of 115 to $119^{\circ}$ at final follow-up. Component
\end{abstract}

\section{R. Schmidt $\cdot$ S. Ogden $\cdot$ A. Alexander}

The Texas Hip and Knee Center Foundation, 750 Eighth Avenue, Suite 400, Fort Worth, TX 76104, USA

\section{J. D. Blaha}

Department of Orthopaedic Surgery, University of Michigan, 2912

Taubman Center, 1500 E. Medical Center Dr., Post Box 5328 , Ann Arbor, MI 48109, USA

\section{A. Fitch}

MicroPort Orthopedics Inc., 5677 Airline Rd., Arlington, TN 38002, USA

C. L. Barnes

HipKnee Arkansas Foundation, 1701 Aldersgate Road, Suite 3, Little Rock, AR 72205, USA

\section{R. Schmidt $(\bowtie)$}

The Texas Hip and Knee Center Foundation, 6301 Harris Pkwy, Suite 300, Fort Worth, TX 76132, USA

e-mail: robert@rschmidtmd.com survivorship, excluding revisions for infection or trauma, was $96.6 \%$ at five years.

Conclusions This study demonstrates subjects implanted with the ADVANCE ${ }^{\circledR}$ Medial Pivot Knee System achieved satisfactory clinical and radiographic midterm outcomes.

Keywords Medial pivot knee $\cdot$ Total knee arthroplasty $\cdot$ Knee kinematics $\cdot$ Knee instability

\section{Introduction}

The "four-bar link theory" of knee kinematics describes the uniform rollback of the femur on the tibia during flexion and for many years has been the accepted model of knee joint motion. When applied to total knee arthroplasty (TKA) implant design, this concept holds that anterior-posterior (AP) motion of the femur on the tibia must occur to avoid "kinematic conflict" and thus conformity between the femoral component and tibial component should be minimized. This theory has led to femoral components with decreasing radii from full extension to flexion and the so-called "J-curve." More recent kinematic analyses of the normal human knee have demonstrated the medial compartment is actually more stable to translation than the lateral resulting in a "medial pivot" motion [1].

The ADVANCE ${ }^{\circledR}$ Medial Pivot Knee System (MicroPort Orthopedics Inc., Arlington, TN, USA) was initially introduced in 1998 by Wright Medical Technology. This system features an asymmetric tibial insert with a highly congruent medial compartment and a less conforming lateral compartment (Fig. 1). Fluoroscopic analysis of this design has confirmed in vivo function consistent with the "medial pivot" philosophy $[2,3]$. Theoretically this design should result in lower tibial insert wear due to its large contact area in the medial compartment and resulting lower contact stresses. 


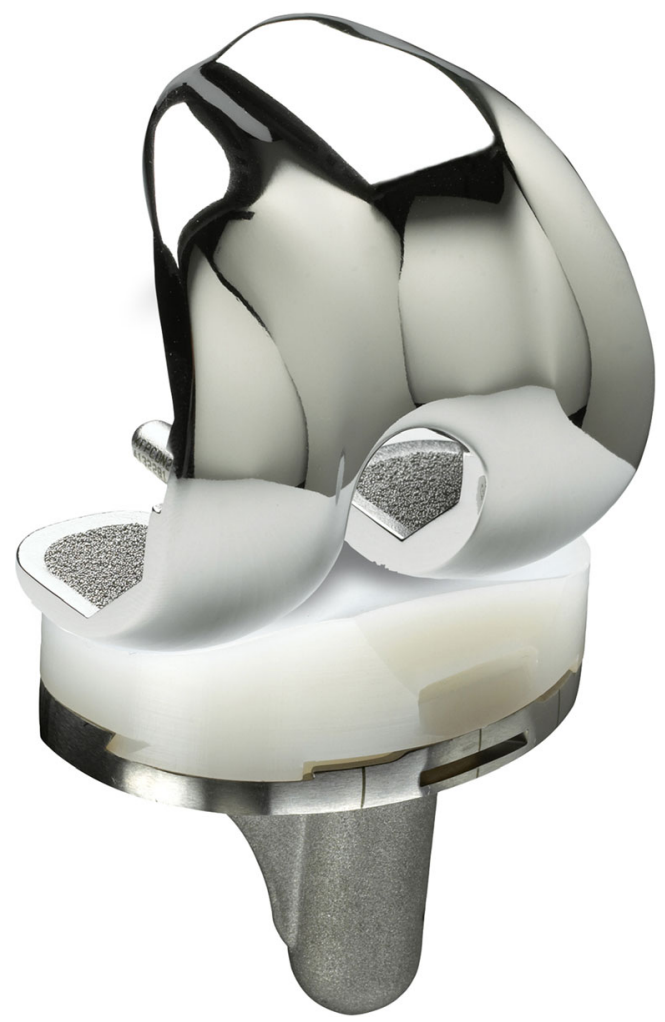

Fig. 1 The ADVANCE ${ }^{\circledR}$ medial pivot knee system (photo provided courtesy of MicroPort Orthopedics Inc.)

Despite this some reports have speculated that the constrained medial compartment could actually result in excessive insert wear or stresses leading to failure [2, 4]. The purpose of this study was to evaluate the midterm clinical and radiographic outcomes of a large cohort of subjects implanted with this system, with particular attention paid to any revisions that could be due to increased tibial insert wear or stresses.

\section{Patients and methods}

Between January 1998 and December 2006, 421 primary TKAs were performed by the senior author (RS) in 373 subjects using the ADVANCE ${ }^{\circledR}$ Medial Pivot Knee System and a surgical technique that resects the posterior cruciate ligament. From 1998 to 2004 implantations were performed using an anterior knee incision and a standard medial parapatellar incision. The anterior exposure was modified in some instances due to existing scar locations from previous knee arthrotomies. After 2004 a less invasive incision was used, beginning approximately $2 \mathrm{~cm}$ proximal to the top of the patella and extending $2 \mathrm{~cm}$ below the joint line. A mini-quad arthrotomy technique was employed and the patella was subluxed and not everted. All other aspects of the surgical technique were the same as those employed prior to 2004 .
Antibiotic administration, thrombosis prophylaxis, and rehabilitation were the same in all subjects.

Subjects were identified from office and hospital records. Any subjects who could not be located were sought through Medicare database and Internet searches. If the subject could not be found using these methods, they were declared lost to follow-up. Available subjects were brought in for a single visit consisting of the Knee Society rating scale, range of motion evaluation, and radiographs $[5,6]$. Another surgeon (SO) at the senior author's practice who had not previously participated in subject follow-up conducted the clinical evaluations. Weight-bearing AP, lateral, and Merchant radiographs were obtained for each TKA. An independent surgeon (CLB) reviewed sequential radiographs to evaluate loosening, subsidence, asymmetry, and radiolucencies using the Knee Society roentgenographic evaluation and scoring system [6].

\section{Statistical considerations}

Knee Society scores and range of motion were reported using mean and standard deviation values. Radiolucencies were reported as the number and size occurring in each zone of interest. Kaplan-Meier analysis was used to calculate component survivorship.

\section{Results}

Of the 373 subjects, 24 (25 TKAs) were lost to follow-up and an additional 29 (31 TKAs) died prior to evaluation. This left a final cohort of 365 TKAs in 320 subjects available for review at a mean follow-up of 5.3 years (2.0-10.9 years). Table 1 shows the demographics for included subjects.

\section{Functional outcomes}

Knee Society clinical scores increased from a mean of 67.1 preoperatively to 95.5 at final follow-up. Scores were categorized using previously reported ranges: excellent $(\geq 90)$, good (89-60), fair (59-35), or poor (<35) [7]. Using this scale there were 324 excellent, 34 good, two fair, and five poor results. The mean range of motion at final follow-up was $119^{\circ}$, compared to $115^{\circ}$ preoperatively.

Table 1 Demographics for included subjects

\begin{tabular}{ll}
\hline Demographic & Value \\
\hline TKAs (subjects), $N$ & $365(320)$ \\
Mean age (range) & 66.5 years $(29-86)$ \\
Male/female & $258(70.6 \%) / 107(29.4 \%)$ \\
\hline
\end{tabular}


Radiographic outcomes

There were no instances of subsidence, osteolysis, or asymmetric narrowing of the tibial insert observed during the radiographic review. In 38 TKAs, there was a $1 \mathrm{~mm}$ or smaller nonprogressive radiolucency in one zone. In six TKAs, there was a $1 \mathrm{~mm}$ or $2 \mathrm{~mm}$ nonprogressive radiolucency in two zones. Three TKAs had radiolucencies in three or more zones. There were no instances of patellar component loosening or osteolysis. The patella tracked normally in 321 TKAs and was tilted, mildly tilted, or mildly subluxed in 26,11 , and five TKAs, respectively. There were no cases of patellar dislocation.

\section{Survivorship analysis}

Kaplan-Meier survivorship was $96.6 \%$ at five years when reoperation for any reason, excluding infection or trauma, was considered as the endpoint. There were seven reoperations: two for infection, three secondary to postoperative traumatic injuries, and two related to component failure. For the two reoperations related to infection, one subject underwent irrigation and debridement with a tibial insert exchange six weeks after index surgery. A second subject required a two-stage revision procedure secondary to a late hematogenous infection after developing a dental abscess. The three reoperations secondary to trauma all involved falls. One subject fell six weeks after discharge, rupturing a patellar tendon, and necessitating the repair of the patellar tendon. The other two subjects fell at one and four years postoperation, respectively. Both sustained secondary ligamentous disruptions that required polyethylene liner exchange and postoperative bracing.

The remaining reoperations were performed for aseptic loosening of the femoral component and possible osteolysis. The subject with possible osteolysis underwent exploratory surgery at another center for a painful knee. The operative report described the implants as stable with a small amount of osteolysis and the development of synovitis, possibly secondary to metal pin wear from the polyethylene insert locking mechanism. The femoral component was revised and the polyethylene liner exchanged. This subject had a history of lumbar sacral disc disease and had marked improvement in the leg pain following a series of epidural steroid injections. Other complications included: six superficial or deep venous thrombosis, six transient peroneal nerve neuropraxia, two non-fatal pulmonary emboli, one intraoperative tibial plateau fracture addressed with internal fixation during the surgery, one haematoma, one early infection, one myocardial infarction, one dysesthesias involving affected foot secondary to a regional block, and 12 delayed healing and superficial wound dehiscence complications that occurred during the initial phase of small-incision surgery before the risks of this technique in subjects with diabetes and rheumatoid arthritis were clarified.

\section{Discussion}

The objective of the present study was to review the midterm clinical and radiographic outcomes of over 350 TKAs performed using the ADVANCE ${ }^{\circledR}$ Medial Pivot Knee System. Ninety-eight percent (98\%) of TKAs experienced excellent or good Knee Society clinical scores. Mean Knee Society clinical scores and range of motion were consistent with scores previously reported for this design $[8,9]$. While a previous study has shown the subject system was preferred by bilateral subjects over other TKA designs implanted in the contralateral knee [10], scores in the present study were not noticeably different than those reported by for other devices. This was expected, as the ceiling effects of the Knee Society score likely prevent it from being sensitive enough to determine if differences exist between TKA designs [11].

Component survivorship was also consistent with rates previously reported for this system in previous studies [12, $8,9]$ and arthroplasty registry reports [13, 14]. A critical factor in component survival is the generation of polyethylene particles due to tibial insert wear. The congruency of the tibial insert's medial compartment and the single-radius femoral component of the subject system should reduce contact stresses throughout the range of flexion and in turn reduce wear. The results of the present study support this hypothesis with radiographic analysis showing no TKAs with evidence of progressive radiolucencies or osteolysis during radiographic follow-up. As mentioned previously, one TKA was presented at another center for suspected osteolysis but was revised before it could be confirmed as part of the present study. All revisions were reviewed for those that could be related to insert wear or stresses (e.g. insert fracture, aseptic loosening, instability). The lone revision for these reasons does not suggest an increased risk with this system. Also supporting this theory is a recent synovial fluid aspiration study in which the size, number, and concentration of polyethylene particles were quantified in subjects implanted with the subject system and posterior-stabilized devices [15]. The investigators reported significantly lower concentrations of wear debris in the medial pivot subjects, suggesting a reduction in wear.

The main limitation of the present study is that the senior author initially only used this device in younger patients with more active lifestyles. As experience with the system increased, he began using it for all subjects except when a well-functioning posterior-stabilized system was already implanted in the contralateral knee. It should be noted the effect of this limitation is likely small. The average age of the current cohort is 66.5 years, which is similar to the average patient age for all TKAs performed in the United Kingdom (69.3 years) [14].

In conclusion, the present study confirms the positive midterm outcomes previously reported for this system $[12,8,9]$. Any concerns over increased revisions for wear or insert 
failure due to the unique insert design appear unwarranted due to the results of this and previous studies. Additional studies are needed to confirm these encouraging results continue at long-term follow-up.

Open Access This article is distributed under the terms of the Creative Commons Attribution License which permits any use, distribution, and reproduction in any medium, provided the original author(s) and the source are credited.

\section{References}

1. Komistek RD, Dennis DA, Mahfouz M (2003) In vivo fluoroscopic analysis of the normal human knee. Clin Orthop Relat Res 410:6981. doi:10.1097/01.blo.0000062384.79828.3b

2. Miyazaki Y, Nakamura T, Kogame K, Saito M, Yamamoto K, Suguro $\mathrm{T}$ (2011) Analysis of the kinematics of total knee prostheses with a medial pivot design. J Arthroplast 26(7):1038-1044. doi:10.1016/j. arth.2010.08.015

3. Schmidt R, Komistek RD, Blaha JD, Penenberg BL, Maloney WJ (2003) Fluoroscopic analyses of cruciate-retaining and medial pivot knee implants. Clin Orthop Relat Res 410:139-147. doi:10.1097/01. blo.0000063565.90853.a4

4. Fan CY, Hsieh JT, Hsieh MS, Shih YC, Lee CH (2010) Primitive results after medial-pivot knee arthroplasties: a minimum 5-year follow-up study. J Arthroplast 25(3):492-496. doi:10.1016/j.arth. 2009.05.008

5. Insall JN, Dorr LD, Scott RD, Scott WN (1989) Rationale of the knee society clinical rating system. Clin Orthop Relat Res 248:13-14
6. Ewald FC (1989) The knee society total knee arthroplasty roentgenographic evaluation and scoring system. Clin Orthop Relat Res 248: 9-12

7. Fuchs R, Mills EL, Clarke HD, Scuderi GR, Scott WN, Insall JN (2006) A third-generation, posterior-stabilized knee prosthesis: early results after follow-up of 2 to 6 years. J Arthroplast 21(6):821-825. doi:10.1016/j.arth.2005.10.008

8. Karachalios T, Roidis N, Giotikas D, Bargiotas K, Varitimidis S, Malizos KN (2009) A mid-term clinical outcome study of the advance medial pivot knee arthroplasty. Knee 16(6):484-488. doi:10. 1016/j.knee.2009.03.002

9. Chinzei N, Ishida K, Tsumura N, Matsumoto T, Kitagawa A, Iguchi T, Nishida K, Akisue T, Kuroda R, Kurosaka M (2014) Satisfactory results at 8 years mean follow-up after ADVANCE(R) medial-pivot total knee arthroplasty. Knee 21(2):387-390. doi:10.1016/j.knee. 2013.10.005

10. Pritchett JW (2011) Patients prefer a bicruciate-retaining or the medial pivot total knee prosthesis. J Arthroplast 26(2):224-228. doi:10. 1016/j.arth.2010.02.012

11. Na SE, Ha CW, Lee CH (2012) A new high-flexion knee scoring system to eliminate the ceiling effect. Clin Orthop Relat Res 470(2): 584-593. doi:10.1007/s11999-011-2203-5

12. Vecchini E, Christodoulidis A, Magnan B, Ricci M, Regis D, Bartolozzi P (2012) Clinical and radiologic outcomes of total knee arthroplasty using the advance medial pivot prosthesis. a mean 7 years follow-up. Knee 19(6):851-855. doi:10.1016/j.knee.2012.04.002

13. Danish Knee Arthroplasty Register (2013) Annual Report. Den Ortopædkirurgiske Fællesdatabase, Aarhus, Denmark

14. National Joint Registry of England, Wales, and Northern Ireland (2013) Annual Report. National Joint Registry, Hemel Hempstead, Hertfordshire, UK

15. Minoda Y, Kobayashi A, Iwaki H, Miyaguchi M, Kadoya Y, Ohashi H, Yamano Y, Takaoka K (2003) Polyethylene wear particles in synovial fluid after total knee arthroplasty. Clin Orthop Relat Res 410:165-172. doi:10.1097/01.blo.0000063122.39522.c2 